\title{
Interactive Application for Visualisation of the Form of Written Postmodern Music
}

\author{
Anna Shvets \\ Plac Marii Skłodowskiej-Curie 5 Lublin \\ Langiewicza 26/12D, 20-032 Lublin \\ Poland \\ annashvets11@gmail.com
}

\begin{abstract}
The contemporary visual epoch with fast software development not only requires scientific data visualisation applications, but also offers possibilities of creation of these applications. Music theory data, especially the structure of music works, acquires a special attention in such a context. The significant application implementation of electronic music structure visualisation was done by the GRM group in the 'Acousmographe' application which presents different kind of texture as graphical figures of different configurations. Martin Wattenberg attempts to analyze the structure of the written music in his 'The Shape of Song' application, where repeated, identical passages of a composition are connected by translucent arches. Such a method works with simple one-line melody data, however, complex structures with polyphonic features need other approaches. The problem that appears in the structure visualisation of music works of professional composers consists in the difficulty of application of some general approach to reach the appropriate form of visualisation of the given structure. In this context, the software application for interactive visualisation of the organ piece 'Mein Weg' of Arvo Pärt was built using the Python programming language. The main idea of this application consists of two general rules. The first rule assumes the simultaneity of visual and audio parts, so the structure could be seen as processing during the music performance. The second rule is based on interactivity of the whole process, so the recipient could manage the process of execution and what appears at the screen. The graphical approach used for the given application is a result of the musicological analysis of the organ piece 'Mein Weg' of Arvo Pärt published by the author in 2012.
\end{abstract}

Music form visualisation. Postmodern music. Interactive application.

\section{INTRODUCTION}

The different forms of music data visualisation appeared in the modern time. All this applications could be classified into two main categories:

- Applications which visualize the physical properties of the sound.

- Applications which try to find the new ways of graphical notation, including interval, tonal, form structures presentation.

The first group could include such programs as Sonic Visualizer (Sonic Visualizer 2011), Raven (Raven 2012), modules of Adobe Audition CS6 (Adobe 2012), Acousmographe (GRM 2011), Interactive Aural Analysis (Clarke 2010) etc. This first group of programs uses audio formats only.

The second group uses mostly MIDI data and could be devised into smaller groups regarding its aims and functionality:
- Alternative notation: Hyperscore (Hyperscore 2010), modules of Cubase (Steinberg 2013), MIDITrail player (MIDITrail 2012) etc.

- Tonal organization: MuSA.RT project by Elaine Chew and Alex François (Chew \& François 2005), hypersphere models of Gérando Baroin (Baroin 2011).

- Accord organization: Isochord (Bergstrom, Karahalios \& Hart 2007).

- Form structure visualisations: The Shape of Song by Martin Wattenberg (Wattenberg 2002), older programs using arc diagrams principle, such as TimeSketch (Chan 2007) or Variations2 (Isaacson 2005).

The most significant research in the field of second type of visualisation was made by Stephen Malinowski and his Music Animation Machine player (Malinowski 2001) containing the tools for visualisation to almost all the presented fields for the second group. 
The analysis of Audio and MIDI entries is quite developed by MIR community (Lartillot 2011). The information about velocity, tune, spectrogram etc. can give the ways to visualize the pitch, rhythm, dynamics, timbre. However the visualisation of the hole structure and find in there the regularities of its creation, using these methods, is quite difficult.

The main problem in the form visualisation consists of the question of how can we teach the computer to recognize the most significant features from all the others to reflect correctly the form structure. This is a question to resolve by the development of artificial intelligence and technologies to come. Nevertheless at the given moment we can consider some logical algorithms of the form creation inherent to the postmodern music.

\section{2. "MEIN WEG" FORM STRUCTURE}

The entire analysis of the organ piece "Mein Weg" by Arvo Pärt and justification of the method in the context of composer's creative process was made in a previous article (Shvets 2012). In this article we'll give just short description of the received form structure and its features to make clear what we are expecting from the visualisation application.

\subsection{Division into 3 layers}

The whole music matter of the piece could be divided into three layers, among the rhythmic structures that were used inside each layer. Such a division is visible from the first measure and reserved by the composer for the entire piece. First layer consist of eights, second of quarters and the third of half notes. Such a division is made hierarchically, so the longer notes correspond to lower layers.

\subsubsection{Tintinnabuli technique of composition and diads inside each layer}

Each layer contains two voices, which are called according to the Arvo Pärt's tintinnabuli technique of composition (Hiller 1997), a Melodic voice (Mvoice) and a Tintinnabuli voice (T-voice). The Melodic voice is independent in its movement and the Tintinnabuli voice, containing only sounds of tonic triad, is subordinated the Melodic voice by a chosen formula. The formulas express the position of the Tintinnabuli voice to the Melodic one. Thus, the connection of the higher nearest tone of Tintinnabuli voice to the tone of Melodic voice is expressed by $T+1$ formula; the lower nearest $T$ tone connected to the M-tone by $T-1$ formula; the second after higher nearest $\mathrm{T}$-tone by $\mathrm{T}+2$ formula and the second lower nearest $\mathrm{T}$-tone by $\mathrm{T}+2$ formula. In the piece that have been analyzed, two higher layers uses $T+1$ formula of T-tone addition and the lowest layer is build according to the $T+2$ formula.

\subsection{Additional logic of the form building}

Principle of addition occurs not only in the way of how the T-voice joins the M-voice, but in the way of how the whole structure is built. Let us mention that the division on the three specified layers corresponds not only to the field of rhythmic, but also because of independent logic of development of each layer. That is the reason why we'll make the visualisation in our application of each layer separately at first and then bind them together layer by layer.

The explanation of the principle of addition in the structure is closely related to the tintinnabuli technique; because the tone of $\mathrm{T}$-voice joined to the M-voice causes the occasional intervals and such an interval from the given range of them becomes the structure to be added for extension of stanzas. The above mentioned range of occurring intervals is set within first octave and that is repeated while graduate extension of stanzas.

\subsubsection{Marks of the end of stanzas. Types of movements inside stanza}

The end of each stanza is marked by an interval of appropriate rhythmic value for the given layer and separated from right and left by the pauses of the same rhythmic value. Thus, the interval of the rhythmic value of eight separated from the left and right sides by eight's pauses serves as a mark to the first layer, quarter with the same logic to the second layer and half note to the third. Each stanza contains two pairs of antagonistic movements, such as movement - anti-movement, mirrored movement - mirrored anti-movement. We may notice that the mark in the second layer will be changed a bit after its first clear appearance. We will see three intervals instead of one separated by the pause only from the left side. The mark, however, will be still recognizable, because it will suddenly return after following interval additions to the same pitch as in the introduction.

\subsubsection{Variables assignment}

After recognition of such a mark behavior it's all naturally to assign the variable "0" to the departing interval in the given layer, because it is always returning back and finally finishes the whole piece. We may suggest that the same logic as we had with T-tone placement expressed in the formula will be appropriate to the assignment of the variables for the other intervals, which come as nearest high or nearest low interval to the " 0 " interval just as shown in Fig. 1. 


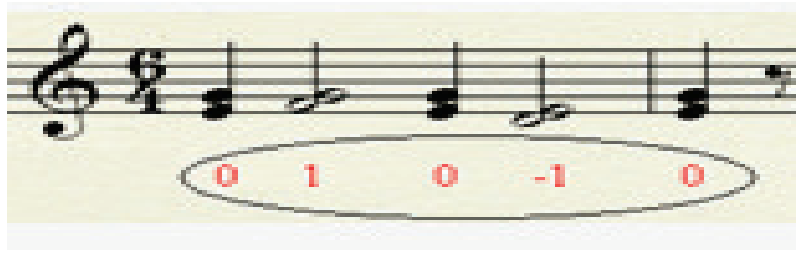

Figure 1: Assignment of variables to the intervals

As we expected, we have received the strict matrices of variables which capture the strict mathematical additions of intervals to stanzas (Table 1):

Table 1: Stanza's extension expressed in variables

$$
\begin{array}{r}
\frac{0,+1,-1,0}{+2,+1,0} \\
-\mathbf{2},-1,0,+1,+2,+\underline{\mathbf{3}} \\
0,-1,-2,-\underline{\mathbf{3}}
\end{array}
$$

$$
\begin{array}{r}
+4,+3,+2,+1,0 \\
-4,-3,-2,-1,0,+1,+2,+3,+4,+\underline{5} \\
0,-1,-2,-3,-4,-\underline{5}
\end{array}
$$

$$
\begin{array}{r}
+6,+5,+4,+3,+2,+1,0 \\
-6,-5,-4,-3,-2,-1,0,+1,+2,+3,+4,+5,+6,+\underline{7} \\
0,-1,-2,-3,-4,-5,-6,-\underline{7}
\end{array}
$$

etc.

The sign " $\{0\}$ " is the mark which ends each stanza. As mentioned before, this mark is the interval, different for each layer. Thus, the mark interval for the first layer is minor third of the tonic triad e-moll $\left(" e^{4 "}-" g\right.$ " $)$, the mark interval for the second layer is perfect forth from the tonic triad (" $\left.h^{3 "}-" e^{4 "}\right)$, and the mark interval for the third layer is perfect fifth $\left(" e^{2, "}-" h^{2 "}\right)$. All these intervals appeared at the very beginning of the piece and were repeated during the two first measures. These initial repetitions are not indicated in the table. From the third measure the diads (connection of M-voice and T-voice) in all the layers begin to move. Let us say that the very first movement containing only four intervals plays the role of initio in the whole form structure. That's why it is separated from the main body of the structure by a line. The first stanza begins already by extension - movement and mirrored movement is extended by one interval $(+2,-2)$ and the following anti-movement with its mirrored version is extended by two intervals $(+3,-3)$.

\subsubsection{Visualisation of received variables using Excel}

Such an extension occurs in all the layers, only the quantity of the added intervals is different and this feature is related to the rhythmic correlations between layers. Nevertheless, the logic of stanzas extension in all the layers will be the same - the two first types of movement extended by one interval and the two last types of movement of each stanza extended by two intervals (including the first added).

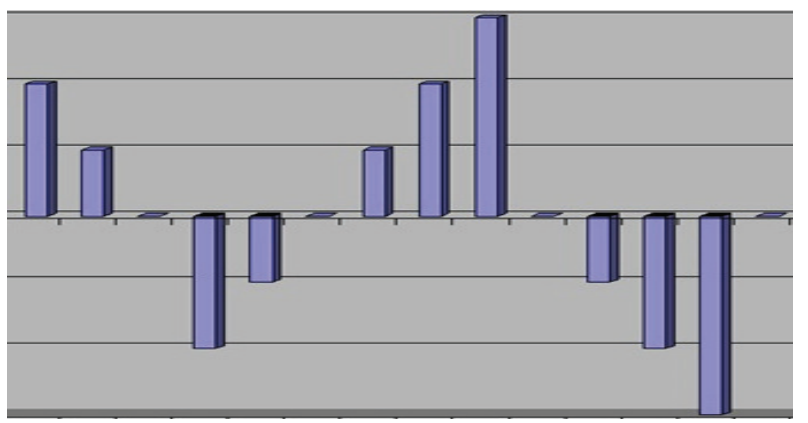

Figure 2: First stanza visualisation using a histogram

The highest, first layer with the smallest rhythmic values reaches the $+15 /-15$ aims in the two last types of movement of the stanza. After this culmination the stanzas begin to be constricted gradually and mathematically exactly using the same logic as with addition of intervals until the primer initio returns back.

The middle, second layer with middle-long rhythmic values reaches only $+9 /-9$ aims in the two last types of movement of the stanza. The same constriction as in the first layer occurs in the second layer, also until the return of primer initio.

Finally, the lowest, third layer with the largest rhythmic values reaches the $+9 /-9$ aims in the two last types of movement of the stanza. The fact that the lowest layer reached the same aims as the middle layer could be rather strange, if we consider that its rhythmic values are two times longer. That happened because the logic of additions in the third layer is not as mathematically exact as in the two higher layers and resembles rather an allusion than a strict following. However, all the four types of movement appear clearly. The constriction is also original in this layer - the mirrored anti-movement figure from -7 to 0 finishes the piece. 


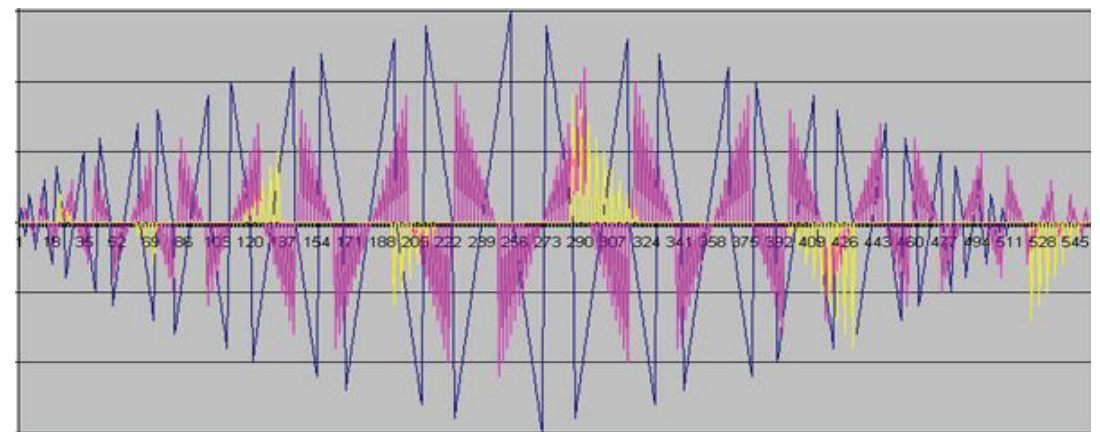

Figure 3: Visualisation of all three layers within the entire piece

Let us take a look to the visualisation of all three layers using the histogram presentation done in Excel (Fig. 3). The histogram in blue color reflects the movement of the first, highest layer, the histogram in rose color reflects the second, middle placed layer and the histogram in yellow - the lowest, third layer. Such visualisation will be the departing point in building of our visualisation application.

The defaults that we may see there is that in the view of the Excel program settings it is impossible to change the color in the same histogram, so we can't see these marks, remaining the end of each stanza. The question of spatialization also will be considered more deeply, because in this visualisation all the three layers are starting at the same position of $x$ and $y$ coordinates. It's logically right, because we assigned variable " 0 " to the first interval appeared in each layer, but as we can see it causes the crossing of histogram. The physical pitch position of the intervals is also different - first interval of the first layer is higher than the first interval of the middle layer and the last is higher than the first interval of the third layer, thus these relations have to be depicted correctly in our visualisation application.

\section{COMPARATIVE ANALYSIS OF "MEIN WEG" VISUALISATIONS}

Let us consider whether other any of known existing program could be useful to the "Mein Weg" form visualisation. Let us start with Music Animation Machine player. First of all we have to choose the corresponding display type to get the information we are looking for. We could be probably interested in the piano roll (MAM), the interval + pitch (YARN) and the shapes views as well as in the three part views (part sequence (LINES), the part motion (BALLS), the part trajectory (WEDGES)). Let us choose the view in the piano roll (MAM) at first and capture in the static image the beginning of the first stanza (Fig.
4). We'll get visualisation synchronized with sound, which is important, however it doesn't disclose the described regularities, because it visualizes too much details, such as duration of each interval (by the length of lines) or the pitch of each note (by the color).

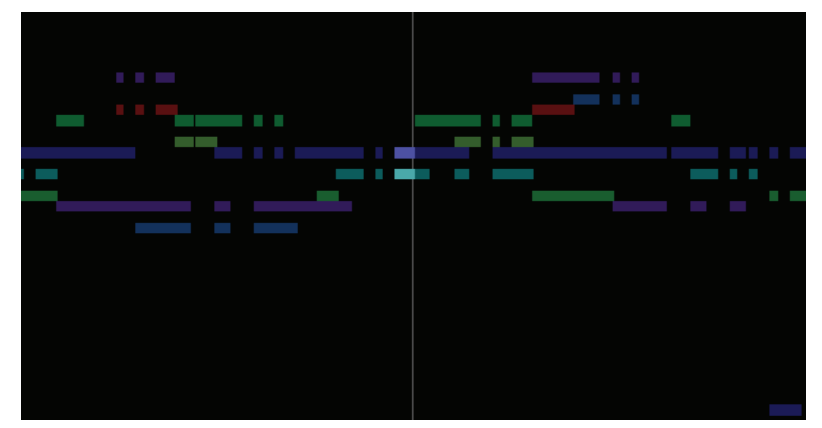

Figure 4: The piano roll (MAM) view

Let us choose less detailed display, such as shapes view. Now we don't see the duration of the notes and the pitch of each note is displayed with different figures of different forms. It's also colored in pitch class mode, but we can choose part (MIDI track/channel) mode to get the white-black visualisation (Fig. 5).

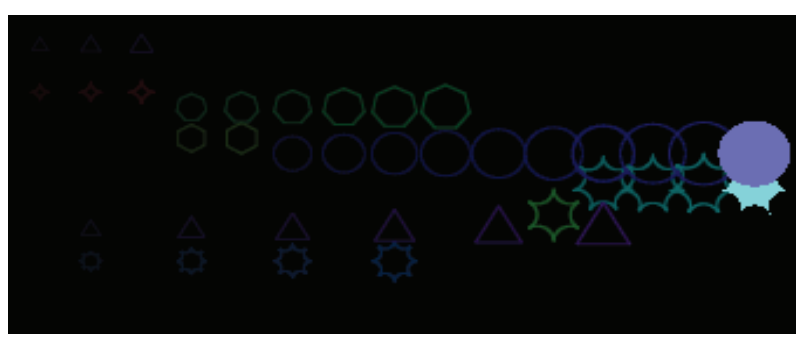

Figure 5: Shapes view 


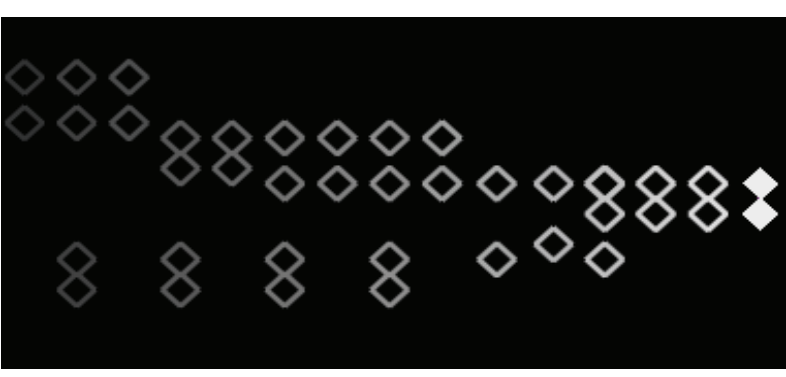

Figure 6: The interval+pitch (YARN) view

The different forms of figures still bring too much of differentiation to such a small part of music.

Trying to find more generalized view let us change the display type to the interval+pitch (YARN) view (Fig. 6). This visualisation displays more meaningfully the motion in stanza, however, as the two previous visualisations, it doesn't give us the panoramic view to capture all the structure form. The three other visualisations using the part view don't resolve this problem either.

\subsubsection{The Shape of Song}

Only the Shape of Song application could probably give us the panoramic view, but once again, it won't display neither the marks nor the existing regularities of addition. The method of arc diagram at all (Wu, Bello 2010) and the algorithm of this application in particular is choosing at least the two neighboring melodic sounds and as we know, the mark which ends each stanza consists of only one interval (one melodic sound), also the visualisation will be too dense - each next stanza includes all the intervals of the previous stanza, even more, each opposite movements of the same stanza contains the same intervals (if not additional intervals). This means that it will be seen by the program algorithm as corresponding melodic segments and joined by translucent arc, but let's remember that the first layer contains 14 stanzas, thus 28 antagonistic pairs with the same ranges of intervals.

\subsubsection{MIDITrailer}

If we use the MIDITrailer player (MIDITrailer, 2011), for example, a MIDI player which provides 3D visualisation of MIDI datasets and which actually allows us the view of the whole structure at the same time, we'll have another problem. This program visualizes notes and works similarly as Music Animation Machine player in the piano roll view (which has been shown in Fig. 4) with such difference that the visualisation is made in threedimensional space and the user can view these 3D objects from different angles. Thus we will have the same problem as with similar visualisation from Music Animation Machine player - it will show us the rhythmic repetitions and blur the contour of the form structure. The function scale allows the user to see the whole structure as one image, but once again, it visualizes the notes, not the form of each layer. While each layer extenseses, the range of intervals (ranges of two notes) actually cross each other and if we use the notes visualisation it will reflect such an intersect. The visualisation we will receive will give us the blurred image of structure: firstly - horizontally (with displayed rhythmic repetitions) and secondly - vertically (with crossed ranges of intervals of the three given layers). Thus, the structure of each layer will not be displayed correctly and we won't get the information we are looking for - the form structure of "Mein Weg" piece of Arvo Pärt.

\subsubsection{Reasons of other application failure}

Let us consider why the existing methods of visualisation don't work with Arvo Pärt's compositions:

- The author's technique tintinnabuli assumes that interval is not independent, but occasional matching of Melodic and Tintinnabuli voices (Hiller 1997). Thus, the use of colors (Klemenc 2008, Ciuha, Klemenc \& Solina 2010) for different intervals visualisation will not give the key to understand the music structures.

- Horizontal thinking with use of diads of Melodic and Tintinnabuli voices, do not allows making an automatic separation into main melodic voice and the accords. Thus, the systems that try to visualize the separate music accords are not applicable (Bergstrom, Karahalios \& Hart 2007).

- Modal thinking: the whole piece is written in the same modal mode - e-moll with variant (moll/dur) third. This fact makes all the tonality visualisation approaches (Mardirossian \& Chew 2007) not useful for music analysis of the given piece.

The kind of polyphony occurs between layers, not melodic lines, thus the methods and applications for music with polyphonic base (Meredith, Lemström \& Wiggins 2003) will not display correctly the form building processes inherent to this piece.

\section{ALTERNATIVE INTERACTIVE APPLICATION}

The lack of appropriate application for form visualisation led the author of the paper to the creation of a new application which focuses on the visualisation of the given piece structure. The application was done using the Python programming language with the use of additional modules. 


\subsection{Functionality}

The main menu consists of three sub-menus: Interval pitch, Harmonic rhythm, Bind layers and Help menu. The Interval pitch menu has three options: Layer 1, Layer 2 and Layer 3 which shows the visualisation of the entire structure of specified layers. The Harmonic rhythm menu contains $1^{\text {st }}$ layer, $2^{\text {nd }}$ layer, $3^{\text {rd }}$ layer options and is intended for a deeper comprehension of occurring form building processes using harmonic rhythm mode view (will be explained further). The Bind layers menu consists of the four options: $1^{\text {st }}+2^{\text {nd }}, 2^{\text {nd }}+3^{\text {rd }}, 1^{\text {st }}+$ $3^{\text {rd }}$ and All layers which actually bind the layers visualisations in chosen connection. More detailed possibilities of these options will be described below. The Help menu provides description documentation for effective use of application.

\subsubsection{Sounding}

The application has two modes of sounding: automatic and manual. Automatic mode is available in Interval pitch and Bind layers options and the manual mode is reserved for the Harmonic rhythm menu. Both are synchronized with visual events and could be changed by pressing the appropriate bottoms visible in the console.

\subsubsection{Labels}

The labels indicating the number value of the added intervals (shown as higher aims) also available as additional option in Interval pitch and Harmonic rhythm menus. The number values are taken from the table which has been shown (Table 1), using described method.

\subsection{Visualisation features}

The idea of the histogram display is conserved in this visualisation too, but to make the comprehension more simple for understanding we have used the blue boxes (seen there as blue squares) to visualize each value. This choice was done under the influence of existing applications for alternative notation, mentioned above, but the main difference consists of that we are not displaying the notes, but the interval height, thus two notes are considered as one, according to the tintinnabuli composition technique, where two voices becomes as one (Tokun 2007, Tokun 2008).

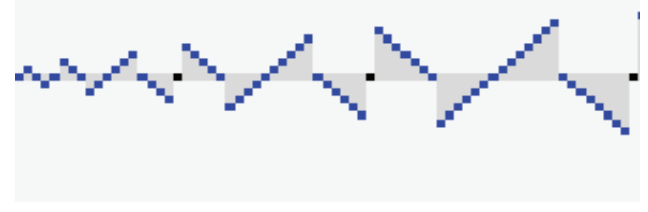

Figure 7: Snapshot of visualisation interface

\subsubsection{Visibility of marks}

The marks, which signify the end of each stanza, are shown as black boxes, very clearly visible on the white background (Fig. 7); in such a way we make the difference between this interval inside stanza structure and at the end, which is impossible in other applications for visualisation that we have tried to apply.

\subsubsection{Music animation}

The basics of music animation are similar to these which are used in the Music Animation Machine, thus each visualized variable is considered as event with appropriate unique numbers of sounds (one interval, repeated or not repeated). Such events appear visually as highlighted aims (boxes) while running of the program.

\subsubsection{Different menus features}

As we mentioned above, the Harmonic rhythm menu allows the user to zoom on the occurring processes. It also allows viewing the form structure of specified layer from different angles while sounding.

\subsubsection{Harmonic rhythm}

While we made the connections between the layers such phenomenon as harmonic rhythm appeared. The time when one repeated interval stays at the same pitch position could be different from the time needed for the neighboring interval. Fortunately the formula of harmonic rhythm is the same for the entire piece, so it's not too complicated, and consists of the $1 / 2$ ratio or $2+4$ formula, where variable "2" is the number of repetitions for one interval and the variable "4" is the number of repetitions for the neighboring interval. All the other harmonic rhythm development consists of this formula continuous repetition. The harmonic rhythm is not changing the general rhythmic distribution and the matter of the $2+4$ formula in the first layer will make the eights, for the second layer the quarters and for the third the half notes.

This is the main reason why we were obliged to visualize the changes between groups of repeated intervals during the simultaneous movement in each layer and then make the correlation between them, which will be displayed in the next part of this topic.

\subsubsection{Spatialization}

The problems of spatialization are resolved in such a way that the different layers have their own space on the canvas and do not cross with each other. Such a resolution allows the user to see structure of each layer independently and, at the same time, to see their relations in the form. Thus, we can see from such visualisation the complementary principle of the layers correlation, the principle of addition in each layer composition, the culmination 
points, which are different for each layer and in such a way the whole structure of the composition. In Fig. 8 below we will show only the beginning of the piece, made from the Bind layers menu, All layers option, due to the limitation of images size in the paper.

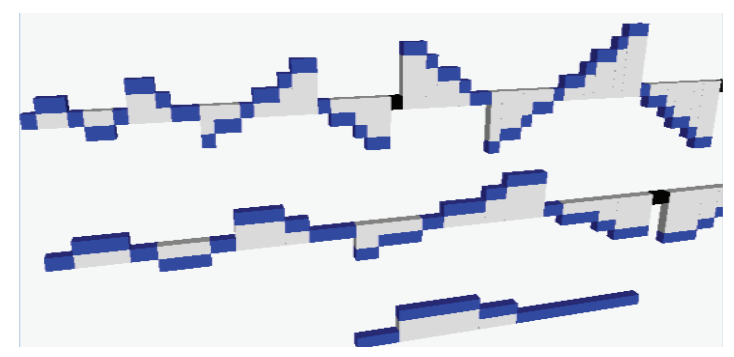

Figure 8: Snapshot of visualisation interface

The presented application may be a part of a bigger one, presenting the form visualisation of the other pieces of Arvo Pärt, which the author of this paper is working on.

\section{CONCLUSION}

We have shown that the visualisation which has been used in the application is correctly reflecting the form of the piece. The principle of musical intervals addition, which extend the length of stanzas is inherent not to the "Mein Weg" piece only, but to the general creativity of the given composer (Zhdanovich 2008, Zamornikova, Katunyan 2011). The assignment of the variables with plus sign to the intervals above the initial interval and with minus sign to the intervals below the initial interval is inherent to the nature of T-tone addition of the tintinnabuli composition technique.

Such an application helps to deeply understand the form building processes of the given piece and could be practically used by musicologists in comparative analysis with the other works of Arvo Pärt, by music teachers who try to explain the bases of the form structure construction in Arvo Pärt's creativity, and also by performers who try to figure out what kind of culminations occurs in the "Mein Weg" piece, especially by the conductors while performing the orchestral transcription of this work. This application could be a module of a bigger program, which will show the structure of the others works of Arvo Pärt and on which the author is currently working on, so the comparative cross analysis of the form structures in the creativity of the given composer could be possible.

\section{REFERENCES}

Baroin, G. (2011) Applications de la théorie des graphes à des objets musicaux: modélisations, visualisations en hyperespace. Thèse de doctorat en musique, Université Toulouse 2, 2011.

Bergstrom T., Karahalios K., and Hart J. (2007) Isochords: visualizing structure in music, Proceedings of Graphics Interface 2007, May 2830, Montreal, Canada, 2007.

Chan, W. W. (2007) A report on musical structure visualization. Department of Computer Science and Engineering at Hong Kong University of Science and Technology, Clear Water Bay, Kowloon, Hong Kong, 2007.

Chew E. and Francois A. (2005) Interactive multiscale visualizations of tonal evolution in MuSA.RT Opus 2. Computers in Entertainment (CIE), 3(4), pp. 1-16.

Ciuha P., Klemenc B., and Solina F. (2010) Visualization of concurrent tones in music with colours. Proceedings of the international conference on Multimedia, Firenze, Italy, 25-29 October, 2010.

Hillier, P. (1997) Arvo Pärt. Oxford, New York.

Isaacson, E. (2005) What you see is what you get: on visualizing music. 6th International Conference on Music Information Retrieval (ISMIR 2005), London, UK, 11-15 September 2005, 389-395. Centre for Digital Music, London.

Klemenc B. (2008) Visualization of music on the basis of translation of concurrent tones into color space, Dipl. Ing. thesis, Faculty of Computer and Information Science, University of Ljubljana, Slovenia, 2008.

Lartillot, O. and Toiviainen, P. (2011) A MatLab Toolbox for musical feature extraction from audio. Proc. of the 10th Int. Conference on Digital Audio Effects (DAFx-07), Bordeaux, France, 10-15 September 2007.

Mardirossian A. and Chew E. (2007) Visualizing music: Tonal progressions and distributions. 8th International Conference on Music Information Retrieval, Vienna, Austria, September 2007.

Meredith, D., Lemström, K. and Wiggins, G. A. (2003) Algorithms for discovering repeated patterns in multidimensional representations of polyphonic music. Cambridge Music Processing Colloquium, Department of Engineering, University of Cambridge, 10 March 2003.

Shvets, A. (2012) "Mein Weg" of Arvo Pärt in the context of postmodernism. Scientific almanac of the Lviv University. Series: Art Studies (in Ukrainian), Issue 11, pp. 31-44. 
Tokun E. (2007) Tintinnabuli: style and technique Musical academy, 2007, № 1, pp. 219-230 (in Russian).

Tokun E. (2008) Tintinnabuli - technique of Arvo Pärt Musicology № 5, 2008, pp. 2-9 (in Russian).

Wattenberg M. (2002) Arc Diagrams: Visualizing Structure in Strings. IEEE Symposium on Information Visualization 2002 (InfoVis 2002), Boston, Massachussets, 28-29 October 2002, 110. IEEE Computer Society Technical Committee on Visualization and Graphics, Boston.

Wu, H. and Bello, J. (2010) Audio-based music visualization for music structure analysis. Sound and Music Computing Conference (SMC), Barcelona, Spain.

Zamornikova K., Katunyan M. (2011) Arvo Pärt's Fratres: The Prayer in Music. Lietuvos muzikologija, t. 12, 2011, pp. 111-136.

Zhdanovich K. (2008), «Tabula Rasa» of Arvo Pärt as significant degree of creativity and example of tintinnabuli - style Scientific almanach of National musical academy of P. Tchaikovsky. Tome 76, 2008, pp. 39-48 (in Ukrainian).
Adobe Audition CS6 (2012).

http://www.adobe.com/products/audition.html (retrieved 29 March 2013).

GRM (2011) Acousmographe.

http://www.inagrm.com/accueil/outils/acousmograp he (retrieved 29 March 2013)

Hyperscore

http://hyperscore.wordpress.com (retrieved 29 March 2013).

Clarke, M. (2010) Interactive Aural Analysis. http://www.hud.ac.uk/research/researchcentres/iaa/ (retrieved 29 March 2013).

Cubase Steinberg (2013).

http://www.steinberg.net/en/products/cubase/start.h

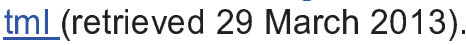

Malinowski, S. (2007) Music animation machine. http://www.musanim.com (retrieved 29 March 2013).

MIDITrail

http://en.sourceforge.jp/projects/miditrail// (retrieved 29 March 2013).

Raven (2012).

http://www. birds.cornell.edu/brp/raven/ravenovervie w.html (retrieved 29 March 2013).

Sonic Visualizer (2012). http://www.sonicvisualiser.org (retrieved 29 March 2013). 